\title{
Opinion
}

\section{Clinical Research: The Future of Medicine Stuck in the Digital Past}

\author{
Elizabeth Del Mastro Benincasa, BA* \\ Manager of Clinical Research, Cordiology Associates of Fairfield County, Connecticut, USA \\ "Corresponding author \\ Elizabeth Del Mastro Benincasa, BA \\ Manager of Clinical Research, Cardiology Associates of Fairfield County, Connecticut, USA; E-mail: elizabeth.delmastro@gmail.com
}

Article information

Received: August 22 ${ }^{\text {nd }}, 2019$; Accepted: September 12 ${ }^{\text {th }}, 2019$; Published: September 19 $9^{\text {th }}, 2019$

\section{Cite this article}

Benincasa EDM. Clinical research:The future of medicine stuck in the digital past. Clin Trial Pract Open J. 2019; 2(I): 17-18. doi: 10.17/40/CTPOJ-2-107

$\mathrm{T}$ The past eight years I've spent working at clinical research sites have been gratifying in countless ways. From seeing direct patient improvement as a result of their involvement in a trial to learning about Food and Drug Administration (FDA) approval of a therapy on which we've conducted trials, the observable outcomes of contributing to the forefront of scientific knowledge are continuously rewarding. However, despite tangible medical field advances, day-to-day operations are often performed in an antiquated fashion. In this article, I highlight four outdated and inefficient methods still utilized in common clinical trial operations and offer suggestions for updated digital replacements.

One of the most time-consuming functions of carrying out clinical trial activities at a research site involves the laborious activity of creating paper source documents to match electronic case report forms (eCRF) in the electronic data capture (EDC) system. In order to appropriately capture the necessary data required in the protocol, the paper source documents must closely, if not exactly, match what is specified in the EDC. While many sites use pre-designed source document templates, every protocol varies drastically in terms of what information is collected and the way in which it is documented, often requiring hours of work and attention to the proper creation and formatting of these forms. Additionally, the eCRF pages are frequently amended in the EDC requiring the paper source to be updated to properly match the requested data. Furthermore, when Clinical Research Associates (CRAs) or monitors come to a site they must verify all paper source documents against what is entered electronically. This not only leaves room for error during the transcription process on the site's end but also creates more arduous work for the CRAs.

The solution I propose for replacing paper source documents is to implement direct entry into the EDC, which very few studies currently employ. This might require sites to be provided with transportable laptops and wireless internet capabilities, however this a small cost to endure when comparing the resources spent creating and then verifying paper source on location. This would reduce the physical space burden of having to store and maintain paper charts on-site and would benefit the sponsor financially by allowing sites to dedicate more time to recruit and conduct patient visits, which would ultimately generate revenue and trial success for both sites and sponsors.

Informed consent forms (ICF) are another realm in which clinical trials often fall behind available technological advances. Potential research subjects are still generally provided with a lengthy paper form that is discussed in detail and signed by the patient, the legally authorized representative (if applicable), and the person conducting the consenting process. A hard copy is provided to the patient while the original wet-ink version is maintained on-site. This wastes costly paper and printer resources for the site and can be more difficult for the patient to read and understand, especially if the type size is small.

I suggest that electronic consent forms be implemented in all trials, allowing the presentation of an ICF on a tablet or laptop to the patient. A paper copy could still be supplied if requested, however resources could be significantly reduced if the site and subject are provided instead with a digital version that could maintained by both parties. A digital version would also enable the text to be magnified for patients who wear glasses or have impaired vision. An electronic consent would also eliminate tracking whether a patient signed the most updated ICF, as a digital log could be easily maintained to confirm the most recent signed version number and inform the site if the subject has not signed an updated ICF either in the form of an alert on the device itself, or through 
an automatic notification to the study coordinator's e-mail. The sponsor or Contract Research Organization (CRO) could also be privy to this information to accurately track which ICF versions have been signed by subjects for internal documentation purposes.

A third way in which clinical trial documentation can be updated is in the form of paper regulatory binders. Regulatory binders, or investigator site files, are kept on location by the site in order to maintain all regulatory documents between the sponsor, CRO, Institutional Review Board (IRB), additional vendors, and the site. Items stored in the binder include, but are not limited to, all start-up documents, contract and budget agreements sponsor communications such as memos and newsletters, and study-specific logs such as subject identification, monitoring visit, investigational product, and site temperature logs.

Most, if not all, of the aforementioned materials, can be digitized and provided to the site via a universal serial bus (USB)-enabled flash drive, which would eliminate the need for cumbersome paper regulatory binders often provided by the sponsor. This would also allow for additional confidentiality measures as electronic signatures can be implemented for official documents such as contract and budget agreements and investigator agreements, and digital copies can be password-protected and maintained digitally. All paper logs could be maintained electronically, as well, eliminating the need to make paper copies or scans for the sponsor trial master file (TMF) and allowing accessibility to the sponsor, CRO, and monitor. On-site verification would also be eliminated or minimized since all logs could be accessed and substantiated electronically through a portal or cloud-based service, reducing the costly and time-consuming need for frequent on-site monitoring visits for data verification.

Finally, another way in which clinical trials are technologically lagging is in the maintenance of inventory of supplies and materials given to the site. For example, sites are generally provided with a pre-specified amount of laboratory kits and shipping materials for each trial utilizing a central lab, which must be reordered manually by the site when inventory is low. This also goes for any other sponsor-provided materials such as retention items given to the subject by the site and investigational product (study drug) inventory.

If sites could digitally mark when lab kits are used or about to expire, and transmit this information directly to the sponsor, CRO, or central lab, it would ensure that sufficient inventory is available on site. Similarly, for retention items and other materials, electronic tracking would eliminate the need for manual reordering and automatically prompt resupply of necessary items. An additional advantage is that the sponsor or CRO would be able to minimize oversupply and only provide the research site with necessary and usable stock thereby eliminating the expensive surplus of medical supplies or ancillary materials that will never be used and must then be discarded by the site.

While the field of clinical research provides ever-evolving additions to medical knowledge, many of the critical operating procedures are still technologically outdated. By employing the four strategies outlined above to eliminate outdated methods by digitally updating key processes, the costly and time-consuming burdens of paper source, paper consent forms, hard copies of regulatory materials, and an under or oversupply of physical materials provided to the site can be greatly reduced. Although some companies are beginning to develop solutions to combat these issues, they have yet to be adopted on a large scale by sites, sponsors, and CROs. Hopefully, as the field catches up with the technological developments utilized in many other industries, clinical trial execution will become more streamlined, more efficient, and more cost-effective for all parties involved. 